\title{
Design and Performance of Brushless Doubly-fed Machine Based on Wound Rotor with Star-polygon Structure
}

\author{
Chaohao Kan \\ College of Electrical \& Automatic Engineering, Hefei University of Technology, Hefei, China \\ Email: kchthw@126.com
}

Received September, 2012

\begin{abstract}
The star-polygon brushless doubly-fed machine (SPBDFM) is a new type of wound-rotor machine and is attractive for variable-speed constant-frequency shaft generation system. The structure of rotor for the machine may be improving the conductor availability of the rotor windings. The kirchhoff laws is employed to illuminate the principle and some combinations of different slots and poles are presented as the examples. Harmonic analysis is also employed to analyze the magnetic motive force (MMF) waveform of rotor winding with the structure of star- polygon. Finally, the results are used to reveal that the SPBDFM is attractive for shaft generation system.
\end{abstract}

Keywords: Polygon; BDFM; Wound Rotor; Magnetic Motive Force

\section{Introduction}

The brushless doubly-fed machine (BDFM), having advanced recently, is a new type AC-excited motor, with the characteristics of synchronous motor and induction motor. There are two sets of symmetrical AC windings in the stator: the control winding with $p_{1}$ pole-pair is connected via a power electronic converter and the power winding with $p_{2}$ pole-pair is connected directly to the grid, and a set of symmetrical AC windings in the rotor. There haven't the direct contact between the two sets of stator windings on voltage or current, but through the couple of rotor magnetic field to realize modulation of two sets of stator rotating magnetic field which have different pole-pair, achieving the motor electrical and mechanical energy conversion and transmission [1-4]. As a generator, the variable-speed constant-voltage constantfrequency sound generation features make its in the wind power generation, marine shaft power generation area; as a motor when the precise timing of its features make it in the higher speed requirements of place has a broad application prospects.

The structure of rotor winding is a key factor affecting the performance of a BDFM, therefore rotor winding needs to be specially designed to achieve the requirement that rotor MMF couple the MMF generated by the stator control winding and power winding [5-8]. When a rotor with $Z_{r}$ bars is subjected to rotating magnetic fields of $p_{1}$ pole-pair, the induced rotor MMF contains main slot spatial harmonic of $p_{1}-Z_{r}$ pole-pair. When $p_{1}-Z_{r}<0$, the rotation direction of the harmonic field is against the main field of $p_{1}$ pole-pair relative to the rotor. Obviously, choose $p_{2}=Z_{r}-p_{1}$ precisely to meet synchronous operation requirement with the BDFM, therefore, the rotor can choose $p_{1}+p_{2}$ slots.

While the sum of stator pole-pair numbers is small, if the number of rotor slots is choice as $p_{1}+p_{2}$, the induced harmonic MMFs of rotor winding is abundant, the leakage reactance may be weaken by increasing the slot number. When the rotor slot number increase to $Z_{r}=$ $K\left(p_{1}+p_{2}\right)$ ( $K$ a positive integer), We have, by the principles of BDFM winding design, each $K$ adjacent rotor slot is a basic unit, which is a minimum slot number group, named the smallest slot number group, also it is known as a phase of rotor winding. Obviously, each smallest slot number group has same winding connection means.

Current the design, it is known as the 'nested-loop' design. As a example, a rotor 54 slots, $1 / 2$ pole-pairs 'nested-loop' is shown as Figure 1. It has 3 nests, and there are 6 loops in each nest.

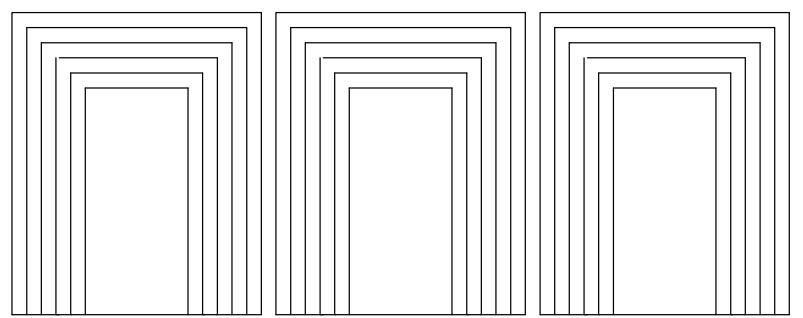

Figure 1. bdfm with rotor 54slots $1 / 2$ - pole pair winding with the connection of nested loop. 
Both the nested loop structure and the reluctance structure of BDFM are "magnetic field" type which by adjusting the flow path to achieve the rotor to couple the magnetic field of the two sets of stator winding. Because of the air gap is sinusoidal alternating magnetic field, rotor winding could not preferable realized coupling two kinds of stator magnetic field only by change the magnetic flux path. the "magnetic field" type structure has defect, such as follows: abundant harmonic MMFs; strong sub-pressure effect; for the relatively small pole-pair number, the winding pitch (nested loop) or the equivalent winding pitch (reluctance) is smaller, especially in the difference between two pole-pair is big, therefore, it is weak that the effect of magnetic coupling for the smaller of the pole-pair number.

In this paper, the rotor winding with "star-polygon" structure of the BDFM is presented. In the novel machine, the "star-polygon" topology is employed, by using this method, the phase current vector of rotor winding will shift a specified angle, and this shift will change the vector of rotor winding MMF, and improve the magnetic field distribution.

\section{Structure and Working Principle of the Rotor}

By Kirchhoff law, it's obviously that the phase current and the line current have a certain phase angle difference in time domain for the connection of polygon circuit. And motor rotor windings also have a certain phase difference in space. If using the space on the phase difference to compensate for the phase difference in time domain, it may be making the two parts of windings MMF generated by superimposed in space, then the windings are star - polygon connection can increase the number of pole-pair corresponding phase windings of the distribution coefficient, thus to improve the utilization of conductors.

To achieve the above functions, the specific winding connection is required. As mentioned above, for the control winding $p_{1}$ pole-pair and the power winding $p_{2}$ pole-pair, the rotor winding is divided into $p_{1}+p_{2}$ phases and each phase winding is divided into two groups. The subscripts $d, s$ denote this two groups. The rotor winding parameters of one phase are defined as follows: $N_{r d}$ is the coil winding number of the polygon part and $N_{r s}$ is the coil winding number of the star part; $W_{r d}$ is each phase winding turns per coil turns of the polygon part and $W_{r s}$ is each phase winding turns per coil turns of the star part. The star-polygon structure of the rotor winding is shown in Figure 2.

As shown in Figure 2, $E_{r d i}$ and $E_{r s i}$ are the ith phase rotor winding induction electromotive force of the polygon part and star part respectively, where $\mathrm{i}=1,2, \ldots$,

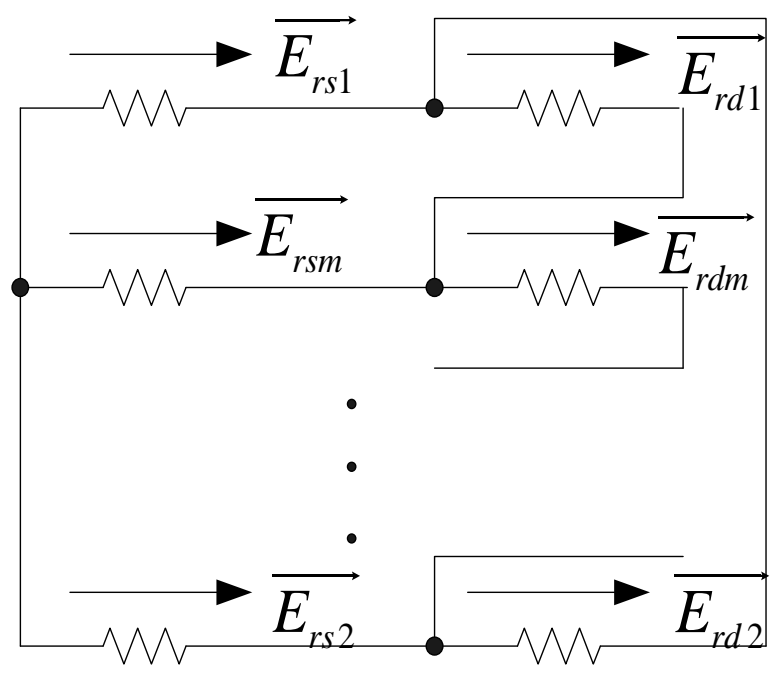

Figure 2. Structure of wound rotor in the form of star- polygon.

$\mathrm{m}$. The number of rotor winding phase is $m=p_{1}+p_{2}$ and $p_{1}<p_{2}$ is supposed.

The principle of the rotor winding with the star-polygon structure is illustrated as follows. When the stator windings of $p_{1}$ pole-pair are imposed with the currents, the gap space will generate the $p_{1}$ pole-pair air-gap magnetic field $[9,10]$. The expression of each phase induced electromotive force for the rotor windings is given by Equation (1).

$$
\left\{\begin{array}{l}
\bar{E}_{r d 1}=E_{r d} \angle 0 \\
\bar{E}_{r d 2}=E_{r d} \angle\left(p_{1} \theta_{1}\right) \\
\vdots \\
\bar{E}_{r d m}=E_{r d} \angle\left((m-1) p_{1} \theta_{1}\right) \\
\bar{E}_{r s 1}=E_{r s} \angle\left(p_{1} \alpha\right) \\
\bar{E}_{r s 2}=E_{r s} \angle\left(p_{1} \theta_{1}+p_{1} \alpha\right) \\
\vdots \\
\bar{E}_{r s m}=E_{r s} \angle\left((m-1) p_{1} \theta_{1}+p_{1} \alpha\right)
\end{array}\right.
$$

where $E_{r d}$ and $E_{r s}$ are each phase induced electromotive force amplitude of the polygon part winding and the star part winding respectively. Since each phase rotor winding is connected in the same manner, the amplitude of the induction electromotive force for the star part and polygon part are equal respectively. $\theta_{1}$ is the mechanical rotor shaft angle. $\alpha$ is the angle between the mechanical axis of the star part and polygon part for one certain phase rotor winding.

It can be seen from the symmetry of topological structure that the current of star part and polygon part are symmetry distribution respectively. Using equal (1) the rotor winding current may be written as 


$$
\left\{\begin{array}{l}
\bar{I}_{r d 1}=I_{r d} \angle \theta_{0} \\
\bar{I}_{r d 2}=I_{r d} \angle\left(p_{1} \theta_{1}+\theta_{0}\right) \\
\vdots \\
\bar{I}_{r d m}=I_{r d} \angle\left((m-1) p_{1} \theta_{1}+\theta_{0}\right) \\
\bar{I}_{r s 1}=I_{r s} \angle\left(-\beta+\theta_{0}\right) \\
\bar{I}_{r s 2}=I_{r s} \angle\left(p_{1} \theta_{1}-\beta+\theta_{0}\right) \\
\vdots \\
\bar{I}_{r s m}=I_{r s} \angle\left((m-1) p_{1} \theta_{1}-\beta+\theta_{0}\right)
\end{array}\right.
$$

where $I_{r d}$ and $I_{r s}$ are the current amplitude which are produced by the polygon part winding and the star part winding of each phase respectively. $\theta_{0}$ is the angle which is between the electromotive force vector and the current vector. By the simplification, we define $\theta_{0}=0$. It's obvious that the angle $\beta=\frac{\pi}{2}-\frac{p_{1} \theta_{1}}{2}$ (see appendix). In order to ensure that the ampere conductors per slot are the same value, the ratio of the current amplitude is $I_{r d}: I_{r s}=W_{r s}: W_{r d}$ approximately.

The axis of polygon part for first-phase winding is counted as the coordinate origin for the space angle. For the $v$ th MMF harmonic may be written as equal (3).

$$
\left\{\begin{array}{l}
f_{r d 1 v}=F_{\phi d v} \cos \left(v p_{1} \theta\right) \cos \left(\omega_{r} t\right) \\
f_{r d 2 v}=F_{\phi d v} \cos \left(v\left(p_{1} \theta-p_{1} \theta_{1}\right)\right) \cos \left(\omega_{r} t-p_{1} \theta_{1}\right) \\
\cdots \\
f_{r d m v}=F_{\phi d v} \cos \left(v\left(p_{1} \theta-(m-1) p_{1} \theta_{1}\right)\right) \cos \left(\omega_{r} t-(m-1) p_{1} \theta_{1}\right) \\
f_{r s 1 v}=F_{\phi s v} \cos \left(v\left(p_{1} \theta-p_{1} \alpha\right)\right) \cos \left(\omega_{r} t+\beta\right) \\
f_{r s 2 v}=F_{\phi s v} \cos \left(v\left(p_{1} \theta-\left(p_{1} \theta_{1}+p_{1} \alpha\right)\right)\right) \cos \left(\omega_{r} t-p_{1} \theta_{1}+\beta\right) \\
\cdots \\
f_{r s m v}=F_{\phi s v} \cos \left(v\left(p_{1} \theta-\left((m-1) p_{1} \theta_{1}+p_{1} \alpha\right)\right)\right) \cos \left(\omega_{r} t-(m-1) p_{1} \theta_{1}+\beta\right)
\end{array}\right.
$$

where $F_{\phi d v}$ and $F_{\phi s v}$ are the pulsating MMFs that are produced by the polygon part winding and the star part winding of each phase respectively. By the ratio of the rotor winding current amplitude $I_{r d}$ and $I_{r s}$, it can be seen that $F_{\phi d v}: F_{\phi s v}=N_{r d} k_{\omega r d v}: N_{r s} k_{\omega r s v}$, where $k_{\omega r d v}$ and $k_{\omega r s v}$ are the polygon part winding and the star part winding of the rotor winding $v$ th harmonic winding factor respectively. $\omega_{r}$ is the frequency of the magnetic field in the rotor reference frame, hence the rotor frequencies in the referred circuit are equal in the magnitude. When $v=1$, the combination of $m$ phase MMFs is

$$
\begin{aligned}
F_{p 1}= & \frac{m F_{\phi d 1}}{2} \cos \left(\omega_{r} t-p_{1} \theta\right) \\
& +\frac{m F_{\phi d 1}}{2} \cos \left(\omega_{r} t-p_{1} \theta+p_{1} \alpha+\beta\right)
\end{aligned}
$$

when $v=p_{2} / p_{1}$, the pole-pair of the combination of phase is $p_{2}$, because the $p_{2}$ pole-pair magnetic field is inverse with the $p_{1}$ pole-pair magnetic field in the ro- tating direction, we have

$$
\begin{aligned}
F_{p 2}= & \frac{m F_{\phi d p 2}}{2} \cos \left(\omega_{r} t+p_{2} \theta\right) \\
& +\frac{m F_{\phi s p 2}}{2} \cos \left(\omega_{r} t+p_{2} \theta-p_{2} \alpha+\beta\right)
\end{aligned}
$$

From equation (4) and (5), it is can be seen that the angle of MMFs which are produced by star part and polygon part of the same rotor phase winding are adjusted as $\beta$. We have

$$
\left.\begin{array}{l}
\beta=\frac{\pi}{2}-\frac{p_{1} \theta_{1}}{2} \\
\theta_{1}=\frac{2 \pi}{m}
\end{array}\right\} \Rightarrow \beta=\frac{\pi}{2}-\frac{p_{1} \pi}{m}=\frac{\pi}{2}-\frac{p_{1} \pi}{p_{1}+p_{2}}>0
$$

Form the equation (4), (5) and (6), it is can be seen that the MMF angle between star part and polygon part of each phase is increased by $\beta$ for $p_{1}$ pole-pair, which is bound to reduce the composite MMFs; and the MMF angle that between star part and polygon part of each phase is reduced by $\beta$ for $p_{2}$ pole-pair, which is bound to increase the composite MMFs. Because $p_{1}<p_{2}$, the decrease in value of $p_{1}$ pole-pair MMF is less than the increase in value of $p_{2}$ pole-pair MMF.

When the three-phase symmetrical voltage sources impose on the $p_{2}$ pole-pair stator windings, it will form $p_{2}$ pole-pair MMF in the air-gap magnetic field, according to the equation $(1) \sim(6)$, and the similar conclusion can be proved: for $p_{1}$ pole-pair, the MMF angle that between star part and polygon part of each phase is increased by $\beta$. For $p_{2}$ pole-pair, the MMF angle that between star part and polygon part of each phase is reduced by $\beta$.

To sum up, the two kinds of air gap magnetic field produced by the $p_{1}$ and $p_{2}$ pole-pair rotor winding are rotating in the opposite direction, and the angle between the magnetic of star part winding and the magnetic of circular part winding will increase while the rotor winding produces the $p_{1}$ pole-pair magnetic field, or reduce while the rotor winding produces the $p_{2}$ polepair magnetic field. Since $p_{1}, p_{2}$ are the fix number, the angle $\beta$ only has one value. If $p_{1}<p_{2}$, the sum of windings distribution coefficients will increase. If $p_{1}>p_{2}$, the sum of windings distribution coefficients of the winding connected in the above mentioned method will reduce. So it doesn't fit for the rotor winding connection.

\section{Prototype Test}

The designed rotor winding has been wound in an YZR225 induction machine, as shown in Figure 3(a). The stator has 72 slots, in which two windings of $p_{1}=1$ and $p_{2}=2$ pole-pair are separately placed. The rotor has 54 slot and its winding connection diagram is shown in Figure 3(b), where y $=15$. 
As a comparison, the rotor winding connection diagram in the general structure is shown in Figure 4.

It is listed in Table 1 that the comparison of harmonic analysis for the resultant MMF produced by three kinds of winding (as shown in Figure3(b), Figure 4 and Figure 1). The coil spans are chosen as 15 slots $(y=15)$ for the former two.

As listed in Table 1, the rotor winding coefficient is decreased by 0.091 as form 1 pole-pair, and the rotor winding coefficient is increased by 0.128 as form 2 polepair. Obviously, the star - polygon structure can improve the overall utilization rate of the rotor windings effectively. Comparable to nested loop winding, the winding coefficient is decreased as form 1 pole-pair, just as form 2 pole-pair. But, the important, the star-polygon connection has significantly smaller in high harmonic MMFs.

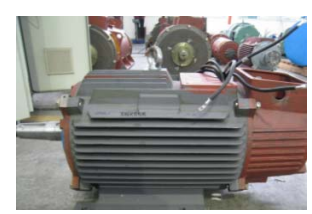

(a) Prototype machine

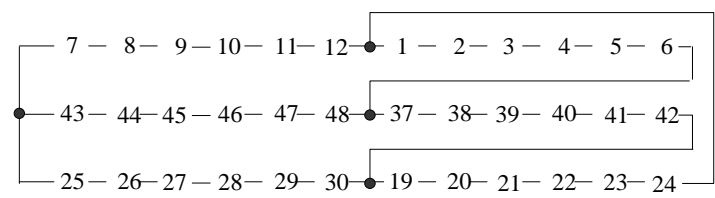

(b) Winding structure

Figure 3. Prototype machine and star-polygon structure of the 54 slots $1 / 2$ pole-pair prototype.

$[1-2-3-4-5-6-7-8-9-10-11-12$
$\square 37-38-39-40-41-42-43-44-45-46-47-48$
$\square-19-20-21-22-23-24-25-26-27-28-29-30-$

Figure 4. A wound-rotor 54 slots and 1/2 pole pairs winding with a general structure.

Table 1. Comparison of the results of MMF harmonic analysis of rotor winding with a structure in the form of star-polygon, general, and nested LooP.

\begin{tabular}{ccccccc}
\hline \multirow{2}{*}{$\begin{array}{c}\text { Pole-p } \\
\text { airs }\end{array}$} & \multicolumn{2}{c}{ star - polygon } & \multicolumn{2}{c}{ general } & \multicolumn{2}{c}{ nested loop } \\
\cline { 2 - 7 } & $\begin{array}{c}\text { Winding } \\
\text { factor }\end{array}$ & $\begin{array}{c}\text { Resultant } \\
\text { MMF } \% \text { ) }\end{array}$ & $\begin{array}{c}\text { Winding } \\
\text { factor }\end{array}$ & $\begin{array}{c}\text { Resultant } \\
\text { MMF }(\%)\end{array}$ & $\begin{array}{c}\text { Winding } \\
\text { factor }\end{array}$ & $\begin{array}{c}\text { Resultant } \\
\text { MMF }(\%)\end{array}$ \\
\hline 1 & 0.615 & -149.4 & 0.706 & -202.7 & 0.642 & -139.8 \\
2 & 0.824 & 100 & 0.696 & 100 & 0.918 & 100 \\
4 & 0.021 & -1.29 & 0.042 & -3.04 & 0.204 & -11.1 \\
5 & 0.047 & 2.28 & 0.093 & 5.37 & 0.255 & 11.1 \\
7 & 0.043 & -1.48 & 0.036 & -1.48 & 0.296 & -9.21 \\
8 & 0.067 & 2.03 & 0.077 & 2.76 & 0.072 & 1.97 \\
10 & 0.055 & -1.33 & 0.063 & -1.80 & 0.008 & -0.18 \\
11 & 0.028 & 0.62 & 0.024 & 0.62 & 0.124 & 2.45 \\
\hline
\end{tabular}

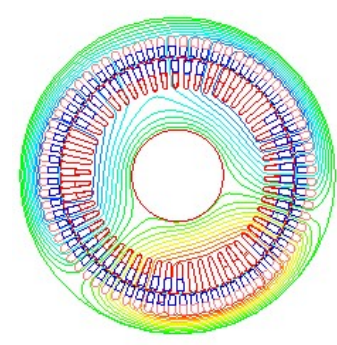

(a) Magnetic field

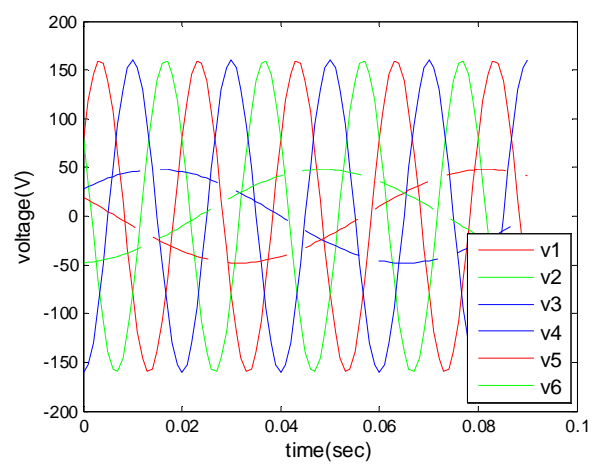

(b) Voltage curve

Figure 5. magnetic field and voltage curve as 2 pole-pair output 5 kw.

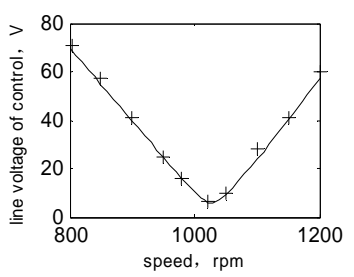

(a) Control voltage

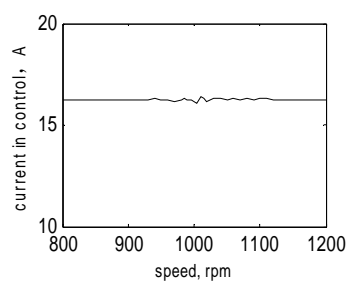

(b) Control current

Figure 6. Control voltage and control current as power winding OUTPUT $5 \mathrm{KW}(\cos \varphi=1)$.

In the experiment, the 2 pole-pair winding is used as the power winding with a line voltage of $200 \mathrm{~V}$ rms (the phase voltage $115.5 \mathrm{~V}$ rms correspond). We keep the power winding output $5 \mathrm{~kW}(\cos \varphi=1)$. As the rotor speed is $1200 \mathrm{rpm}$, the magnetic field is shown as in Figure 5(a); the power winding voltage (named v1, v2 and v3) and control voltage (named v4, v5 and v6) curve are shown as in Figure 5(b) based on FE analysis. As the 
rotor speed is from 800 to $1200 \mathrm{rpm}$, the control voltage vs. speed is shown in Figure 6(a); the control current vs. speed is shown in Figure 6(b).

As shown in Figure 6(a), the experimental data are consistent with the calculated results based on FE analysis. Obviously, the star-polygon BDFM can realize the function of variable-speed constant-voltage constantfrequency generation.

\section{Conclusions}

This paper has presented, for the first time, a new type rotor winding connection, named star-polygon, for BDFM. The BDFM with "star- polygon" structure of rotor windings has the following five characteristics: 1 , It is increased that the rotor winding distribution coefficient which corresponding to the stator power windings; 2, A set of rotor slot conductors is used repeatly, improving the actual utilization of the rotor slot conductors; 3 , The choice of winding pitch is freedom; 4, The connection of rotor winding is flexible; 5, The content of harmonic MMFs is low. So this new type structure of rotor windings has great development prospects.

\section{Acknowledgements}

This work was supported by the Surface of Natural Science Fund Program projects of Anhui Province (NO.12 08085ME62), by the Doctoral Degree in Special Research Fund Program projects of hfut (NO. 2011HGBZ0935).

The authors wish to thank the China Changjiang National Shipping Group Motor Factory for the provision of the prototype machine and fabrication of the rotor.

\section{REFERENCES}

\section{Appendix}

The parameters compute of $\beta$ :

The current vectors, such as $\bar{I}_{r d 1}, \bar{I}_{r d 2}$ and $\bar{I}_{r s 1}$, intersect at the same node, by Kirchhoff current law, we have $\bar{I}_{r d 2}+\bar{I}_{r s 1}=\bar{I}_{r d 1}$, As shown in Figure 7 .

Because of $\left|\bar{I}_{r d 1}\right|=\left|\bar{I}_{r d 2}\right|$, we have $\gamma=\frac{\pi-p_{1} \theta_{1}}{2}$. It is can be seen from Figure 7 that $\beta=\gamma$. So the $\beta$ is given as $\beta=\gamma=\frac{\pi}{2}-\frac{p_{1} \theta_{1}}{2}$. As the rotor windings of each
[1] P. C. Roberts and R. A. McMahon, "Performance of BDFM as Generator and Motor, IEE Proceedings, Electric Power Application, Vol. 153, No. 2, 2006, pp. 289-299.

[2] S. Williamson, A. C. Ferreira and A. K. Wallace, "Generalized Theory of the BDFM. Part 2: Model Verification and Performance," IEE Proceedings- Electric Power Application, Vol. 144, No. 2, 1997, pp. 123-129.

doi:10.1049/ip-epa:19971052

[3] A. K. Wallace, P. Rochelle and R. Spee, "Rotor Modeling and Development for BDFMs," Conference of Record of the International Conference on Electrical Machines, Cambridge, Vol. 1, 1990.

[4] X. Wang and P. C. Roberts, "Optimization of Bdfm Stator design Using an Equivalent Circuit Model and a Search Method,” In Proc, IEE 3th Int. Conf. Power Electronics, Machines and Drives, Dublin, Ireland, 2006, pp. 606-610.

[5] X. Wang, R. A. McMabon and P. J. Tavner, "Design of the Brushless Doubly-fed (induction) Machine," IEEE Interaction Election Machines \&Drives Conference, IEMDC’07, Vol. 2, 2007, pp.1508-1513.

[6] X. F. Wang, “A New BDFM with a Wound-rotor Changing-pole Winding," Proceedings of the CSEE, Vol. 23, No. 6, 2003, pp. 108-111.

[7] S. C. Yang, "Feature of Electromagnetic Design for BDFMs,” Proceedings of the CSEE, Vol. 21, No. 7, 2001, pp. 107-110.

[8] F. G. Zhang, F. X. Wang and Z. Wang, "Comparative Experiment Study on the Performance of Doubly-fed)," Proceedings of the CSEE, Vol. 22, No. 4, 2002, pp. 52-55.

[9] C. H. Kan and X. F. Wang, "Harmonic Anslysis for Wound-rotor Winding in Induction Manchine," Large electric manchine and hydraulic turbine., Vol. 4, 2007, pp. 18-23.

[10] S. Z. Xu, "Windings Theory of A.C. Machines," Publishing House of Machinery Industry, 1985.

phase are symmetry, the value of $\beta$ is not a result of the chosen reference point of change.

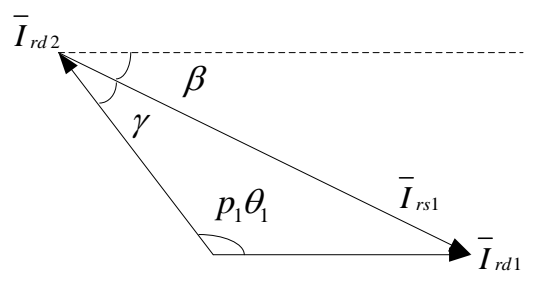

Figure 7. The vector of current. 